\title{
Primary Ovarian Leiomyosarcoma: A Case Report and Review of the Literature
}

\author{
(D) Nergis Kender Ertürk1, (D) Ruken Dayanan², (1) Kadir Çetinkaya2, (1D Cemal Reşat Atalay² \\ 1 University of Health Sciences Turkey, Bursa Yüksek Ihtisas Training and Research Hospital, Clinic of Obstetrics and Gynecology, Bursa, Turkey \\ ${ }^{2}$ Ankara Numune Training and Research Hospital, Clinic of Obstetrics and Gynecology, Ankara, Turkey
}

\section{Abstract}

Ovarian sarcomas represent less than $3 \%$ of all ovarian tumors. Leiomyosarcoma (LMS) is a rare subtype, accounting for only $0.1 \%$ of all ovarian sarcomas. We present a case of primary ovarian LMS in a 68-year-old multigravid woman. She was treated by total abdominal hysterectomy with bilateral salpingo-oophorectomy, followed by adjuvant chemotherapy. The prognosis of ovarian LMS is very poor. Although different treatment modalities like adjuvant chemotherapy and radiotherapy have been described, no clear benefit has been proven.

Keywords: Primary ovarian, leiomyosarcoma, sarcoma

\section{INTRODUCTION}

Primary ovarian sarcomas are rare gynecological tumors accounting for less than 3\% of all ovarian tumors (1). Only a few cases of fibrosarcoma, leiomyosarcoma (LMS), carcinosarcoma, angiosarcoma and other histological types have been reported. Less than $0.1 \%$ of ovarian sarcomas are LMS (2-4). Because ovarian LMS are rare, there are not many case reports in literature, and no prospective studies for management recommendations. Therefore, optimal treatment guidelines have not yet been developed.

There is no treatment modality other than surgery, total abdominal hysterectomy (TAH) and bilateral salpingooophorectomy (BSO) for ovarian LMS (5). They are generally highrisk cancers and have a very poor prognosis. The type and utility of adjuvant therapy is controversial (6).

Here, we present a case of primary ovarian LMS in a postmenopausal woman presenting a giant abdominal mass.

\section{CASE PRESENTATION}

A 68-year-old multigravid woman presented to the gynecology clinic with complaints of abdominal pain and a palpable abdominal mass. Additionally, the patient had a history of hypertension. The patient's pregnancy history was G5 P5, her menarche age was 13 years, and her last menstrual period was 8 years ago, she was postmenopausal.

Physical examination revealed a mobile, hard mass of about 20 $\mathrm{cm}$ in diameter, which was palpable to the level of the umbilicus.

On abdominal ultrasonography, a 120x180 mm multilobulated cystic mass containing many septas and thick walls was seen posterior to the uterus; the origin of the mass could not be detected. Laboratory findings showed tumor (CEA, CA 199, CA 125, CA 15-3) and biochemical markers within normal limits. No malignancy was observed in cervical cytology, and histopathological result of endometrial sampling revealed atrophic endometrium.

Phone: +90 5335152585 E-mail: nergiskender@gmail.com ORCID ID: orcid.org/0000-0002-2902-9670

Cite this article as: Kender Ertürk N, Dayanan R, Çetinkaya K, Atalay CR. Primary Ovarian Leiomyosarcoma: A Case Report and Review of the Literature. Eur Arch Med Res 2020;36(3):226-8

๑Copyright 2020 by the University of Health Sciences Turkey, Prof. Dr. Cemil Taş̧ığlu City Hospital

European Archives of Medical Research published by Galenos Publishing House. 
The patient was scheduled for surgery with a prediagnosis of degenerative leiomyoma. Intraoperatively, a solid cystic mass of about $20 \mathrm{~cm}$ in diameter arising from the left adnex adhered to transverse colon and left pelvic side wall. There was no evidence of right-sided and upper abdominal diseases, and no ascites. Cytology was taken by peritoneal lavage. Adhesiolysis with sharp dissection, TAH and BSO were performed. Intraoperative pathology consultation (frozen section) was demanded, and histopathological evaluation reported a mesenchymal tumor, which could not be distinguished as benign or malignant.

There were no abnormal findings for the first two postoperative days. A day later, the patient complained of abdominal distension, vomiting and nausea. Oral intake was stopped for two days and decompression with a nasogastric tube was done. On the fifth postoperative day, oral regimen was reinstated, and the patient's complaints regressed. She was discharged problemfree one week after surgery.

The histopathological result was left adnexal LMS. Macroscopically, the mass was gray-white, $20 \times 13 \times 11 \mathrm{~cm}$ in size, fibrillar in structure with nodular areas, and multilobulated. Microscopically, coagulative tumor necrosis, $>10$ mitosis per 10 high-power-fields (HPF), diffuse and severe atypia were seen. Cytological evaluation reported benign peritoneal cytology.

At the first month follow up examination, she had no complaints and no lesion was observed on abdominal computed tomography and positron emission tomography scan. Her CA 125 value was $12 \mu / \mathrm{mL}$, and other tumor markers were equally within normal limits. Based on these results, the patient was referred to the department of medical oncology for consultation. They recommended four cycles of adriamycin chemotherapy.

\section{DISCUSSION}

Smooth muscle tumors of the ovary are uncommon, accounting for less than $1 \%$ of all ovarian tumors. These tumors originate from hilar blood vessels or smooth muscle metaplasia of ovarian stromal or theca cells (7). The most common primary ovarian sarcomas are fibrosarcomas, endometrial stromal sarcomas, carcinosarcomas and rhabdomyosarcomas (3). Primary pure ovarian LMS is a very rare tumor (8). In literature, there are some cases mentioned as ovarian LMS. However, the case series are few. Lerwill et al. (7) reported 54 ovarian smooth muscle tumors including 26 LMS.

Ovarian LMS occur typically in postmenopausal women, as in our case. Zygouris et al. (8) calculated a mean age of 52.6 years and He et al. (9) of 54.9 years. Despite this, several cases have been seen in adolescence $(10,11)$.

Ovarian LMSs do not have specific symptoms. Patients may present with abdominal pain, weakness, postmenopausal bleeding, or a palpable abdominal mass, as in our case. The tumor is mostly seen as solid masses with or without cystic degeneration, based on imaging modalities.

Cases of primary ovarian LMSs are so rare that no recommended guidelines on optimal treatment have been established. The main treatment type is surgery which extends from fertility sparing to debulking surgery, consisting of TAH, BSO and excision of the tumoral masses $(5,12)$. Although surgery was performed for all cases, the scope of surgery is controversial. Because the probability of occult metastatic disease is likely low, a second operation is not necessary for patients without lymph node dissection or omentectomy (5). There are different approaches in terms of surgery. In one instance, a 27-year-old patient who underwent complete surgical staging, followed by 6 cycles of gemcitabine-adriamycin chemotherapy had no evidence of recurrent disease over a period of 47 months (13). There are also cases in which TAH + BSO is performed, and adjuvant chemotherapy is given $(14,15)$. In our case we performed TAH + BSO, no per operative palpable lymphadenopathy was found, postoperative imaging were normal, and histopathological findings were compatible with ovarian LMS. This was made of tumor cell necrosis, 23 mitosis per $10 \mathrm{HPF}$, and significant atypia microscopically (7). Immunohistochemical markers like desmin, vimentin, and smooth muscle actin could also be used in the diagnosis of ovarian LMS $(1,6)$.

The prognosis of ovarian LMS is very poor. Although treatment modalities such as chemotherapy and radiotherapy have been described, there is no sufficient data to prove that such treatment will improve survival outcomes (5). The prognosis depends on the tumor size, stage and mitotic index (6). There are no prospective data supporting the routine use of adjuvant chemotherapy for disease limited to ovary (5). In our case, since the mitosis rate was high and the tumor size was large, the oncology department decided on adjuvant chemotherapy. Four courses of single agent (adriamycin) chemotherapy were planned. However, there is no evidence that chemotherapy will improve survival outcome. In our patient, the expectation of disease recurrence is high, and resection of recurrent tumor is recommended (5).

\section{CONCLUSION}

Ovarian leiomyosarcomas are rare tumors and have no established standard treatment. Surgery is thought to be the 
main modality for treatment. Although chemotherapy and radiation therapy are used as adjuvants, there is no strong evidence that they have any additional benefits.

\section{Ethics}

Informed Consent: Consent form was filled out by all participants.

Peer-review: Externally peer-reviewed.

\section{Authorship Contributions}

Concept: N.K.E., Design: N.K.E., K.C.., Data Collection or Processing: N.K.E., R.D., Analysis or Interpretation: N.K.E., K.C,., C.R.A., Literature Search: R.D., Writing: N.K.E., K.Ç.

Conflict of Interest: No conflict of interest was declared by the authors.

Financial Disclosure: The authors declared that this study received no financial support.

\section{REFERENCES}

1. Dixit $S$, Singhal S, Baboo HA, Vyas RK, Neema JP, Murthy $R$, et al. Leiomyosarcoma of the ovary. J Postgrad Med 1993;39:151-3.

2. Bouie SM, Cracchiolo B, Heller D. Epithelioid leiomyosarcoma of the ovary. Gynecol Oncol 2005;97:697-9.

3. Rauh-Hain JA, Del Carmen MG. Carcinosarcoma of the Ovary. In: Uncommon. Gynecologic Cancers 2014;109-19.

4. Anderson B, Turner DA, Benda J. Ovarian sarcoma. Gynecol Oncol 1987;26:183-92.

5. Hensley ML, Barrette BA, Baumann K, Gaffney D, Hamilton AL, Kim JW, et al. Gynecologic Cancer InterGroup $(\mathrm{GCIG})$ consensus review: uterine and ovarian leiomyosarcomas. Int J Gynecol Cancer 2014;24(9 Suppl 3):61-6.

6. Tașkin S, Tașkin EA, Uzüm N, Ataoğlu O, Ortaç F. Primary ovarian leiomyosarcoma: a review of the clinical and immunohistochemical features of the rare tumor. Obstet Gynecol Surv 2007;62:480-6.

7. Lerwill MF, Sung R, Oliva E, Prat J, Young RH. Smooth muscle tumors of the ovary: a clinicopathologic study of 54 cases emphasizing prognostic criteria, histologic variants, and differential diagnosis. Am J Surg Pathol 2004;28:1436-51

8. Zygouris D, Androutsopoulos G, Grigoriadis C, Arnogiannaki N, Terzakis E. Primary ovarian leiomyosarcoma. Eur J Gynaecol Oncol 2012;33:331-

9. He M, Deng YJ, Zhao DY, Zhang Y, Wu T. Synchronous leiomyosarcoma and fibroma in a single ovary: A case report and review of the literature. Oncol Lett 2016;11:2510-4.

10. Monk BJ, Nieberg R, Berek JS. Primary leiomyosarcoma of the ovary in a perimenarchal female. Gynecol Oncol 1993;48:389-93.

11. Saïm M, Limam W, Meatchi T, Ferrand J, Truc JB, Sibony O. Primary ovarian leiomyosarcoma in perimenarche. J Gynecol Obstet Biol Reprod (Paris) 2007;36:306-9.

12. Vijaya Kumar J, Khurana A, Kaur P, Chuahan AK, Singh S. A rare presentation of primary leiomyosarcoma of ovary in a young woman. Ecancermedicalscience 2015;9:524.

13. Nazneen S, Kumari A, Choudhary V, Kumari S, Pankaj S. Prolonged Survival of a Young Female with High Grade Pleomorphic Leiomyosarcoma of Ovary Without Recurrence. J Obstet Gynaecol India 2016;66(Suppl 2):639-41.

14. Nicòtina PA, Antico F, Caruso C, Triolo O. Primary ovarian leiomyosarcoma. Proliferation rate and survival. Eur J Gynaecol Oncol 2004; $25: 515-6$

15. Khabir A, Boudawara T, Ayadi L, Kharrat M, Kharrat M, Beyrouti I, et al Léiomyosarcome ovarien bilatéral de type épithélioïde: une observation [Epithelioid bilateral ovarian leiomyosarcoma: a study]. Ann Pathol 2003;23:47-9. 